\title{
Road-friendliness of Fuzzy Hybrid Control Strategy Based on Hardware-in-the-Loop Simulations
}

\author{
Tian Yi Yan ${ }^{1 *}$ Qiang Li $^{2}$, Kun Ru Ren, Yu Lin Wang ${ }^{1}$, Lu Zou Zhang ${ }^{1}$ \\ ${ }^{1}$ Dept. of Vehicle Engineering, Qingdao University, China, ${ }^{2}$ China National Heavy Duty \\ Truck Group Taian Wuyue Special Vehicle Co., Ltd., China
}

Received: May $26^{\text {th }}, 2012$; Revised: June $22^{\text {th }}, 2012$; Accepted: June $29^{\text {th }}, 2012$

\section{Abstract}

Purpose: In order to improve road-friendliness of heavy vehicles, a fuzzy hybrid control strategy consisting of a hybrid control strategy and a fuzzy logic control module is proposed. The performance of the proposed strategy should be effectively evaluated using a hardware-in-the-loop (HIL) simulation model of a semi-active suspension system based on the fuzzy hybrid control strategy prior to real vehicle implementations. Methods: A hardware-in-the-loop (HIL) simulation system was synthesized by utilizing a self-developed electronic control unit (ECU), a PCI-1711 multi-functional data acquisition board as well as the previously developed quarter-car simulation model. Road-friendliness of a semi-active suspension system controlled by the proposed control strategy was simulated via the HIL system using Dynamic Load Coefficient (DLC) and Dynamic Load Stress Factor (DLSF) criteria. Results: Compared to a passive suspension, a semi-active suspension system based on the fuzzy hybrid control strategy reduced the DLC and DLSF values. Conclusions: The proposed control strategy of semi-active suspension systems can be employed to improve road-friendliness of road vehicles.

Keywords: Road-friendliness, Fuzzy Hybrid Control Strategy, Semi-active suspension systems, Hardware-in-the-Loop Simulations

\section{Introduction}

Magneto rheological (MR) fluids exhibit large reversible changes in their rheological behavior when subjected to external magnetic fields. This crucial property has triggered tremendous research activities in the development of advanced suspension systems. Semi-active suspensions based on MR fluids consume much less power compared with fully active suspension systems.

For improving ride quality, many control strategies were designed for semi-active suspension systems. Fuzzy control theory was applied by Nicolás et al. (1997) to the design of semi-active suspension systems for improving ride quality. It was found that the proposed controller could achieve similar performances to those obtained

\footnotetext{
*Comesponding author: Tian Yi Yan

Tel: +86-532-8595-3676; Fax: +86-532-8595-3676

E-mail: yan_7012@126.com
}

with skyhook-type algorithms, but using a far less expensive sensorization. Field tests of a semi-active suspension system were carried out by Choi et al. (2001), in which four independent skyhook controllers associated with semi-active dampers are employed for better ride quality.

Some regulations for controlling road-friendliness of heavy vehicles have been introduced by several western countries such as United Kingdom, Australia, etc. Moreover, these countries also provide a payload incentive for vehicles equipped with suspension systems having improved road-friendliness. Furthermore, fundamental researches related to road-friendliness of heavy vehicles are supported by the above governments and their automobile companies (OECD, 1998). Due to the limited performance of traditional passive suspensions, researches on road-friendliness of semi-active suspension systems are being continuously conducted to reduce dynamic tire 
force generated by heavy vehicles (Yi and Hedrick, 1989; Yan et al., 2007; Kortüm et al., 2002; Guglielmino et al., 2008). For instance, a full heavy vehicle model with a semi-active suspension system was designed using Simpack software in the EU project called 'Copernicus of SemiActive Damping of Truck Suspensions and Its Influence on Drivers and Road Loads (SADTS)' on vehicle-road interaction to study its road-friendliness (Valášek et al., 1998).

The practical use of semi-active suspension systems is relatively difficult by its inherently hysteretic, time-variant properties. Furthermore, the development of an accurate dynamic vehicle model that includes all relevant factors is almost impossible, because of the complex non-linear dynamics of many important components. All above mentioned nonlinearity and uncertainty resulted in difficult implementation of many model-based control approaches (Caponetto et al., 2003).

The design of model-free control strategy considering the road-friendliness is becoming an important research area of semi-active suspension systems. Therefore, the main objective of this paper is set to develop a model-free fuzzy hybrid control strategy of semi-active suspension systems for improving road-friendliness. Moreover, hardware-in-the-loop (HIL) simulations were conducted in order to evaluate the influence of the time-delay factor on road-friendliness of the proposed control strategy using Dynamic Load Coefficient (DLC) and Dynamic Load Stress Factor (DLSF).

\section{Design of a fuzzy hybrid control strategy}

The Fuzzy hybrid control strategy was designed to improve the overall vertical performance of semi-active suspension systems of heavy vehicles. The proposed control strategy consists of a hybrid control strategy, and a fuzzy logic control module for automatically adjusting the ratio between skyhook control and groundhook control (Fig. 1). The frame of the proposed control strategy is based on the hybrid control approach, which can be formulated as follows (Goncalves , 2001).

$$
\begin{array}{ll}
\left.f_{\text {sa }}=G\left[\alpha \delta_{\text {sky }}+(1-\alpha) \delta_{\text {gnd }}\right)\right] \\
\delta_{\text {sky }}=V_{1} & \text { if } V_{1} V_{12} \geq 0 \\
\delta_{\text {sky }}=0 & \text { if } V_{1} V_{12}<0
\end{array}
$$

$$
\begin{array}{ll}
\delta_{\text {gnd }}=V_{2} & \text { if }-V_{2} V_{12} \geq 0 \\
\delta_{\text {gnd }}=0 & \text { if }-V_{2} V_{12}<0
\end{array}
$$

Where, $\delta_{\text {sky }}$ is the skyhook component damping force, $\delta_{\text {gnd }}$ is the groundhook component damping force, $V_{1}$ is the absolute velocity of the sprung mass, $V_{2}$ is the absolute velocity of the sprung mass, $V_{12}$ is the relative velocity of the sprung mass with respect to the unsprung mass, $\alpha$ is a relative ratio between skyhook control and groundhook control, $G$ is a constant gain.

A fuzzy logic control module shown in Fig. 2 is the core of the proposed fuzzy hybrid control strategy, and its critical role is to intelligently adjust the relative ratio in a real-time manner in order to improve road-friendliness. It's obvious that the relative ratio $\alpha$ is an important factor. In this study, the automatic determination of the relative ratio $\alpha$ depends on the vertical motions of sprung and unsprung masses. For designing of the fuzzy control logic module, the vertical velocity $\boldsymbol{V}_{1}$ of the sprung mass, the vertical velocity $\boldsymbol{V}_{2}$ of the unsprung mass, and the relative velocity between sprung mass and unsprung mass $\boldsymbol{V}_{12}$ were chosen as three fuzzy variables. The fuzzy variables $\boldsymbol{V}_{1}$ and $\boldsymbol{V}_{12}$ were selected as input variables of the fuzzy control logic module, the following linguistic input variables are selected to describe fuzzy variables $\boldsymbol{V}_{1}$ and $\boldsymbol{V}_{12}$.

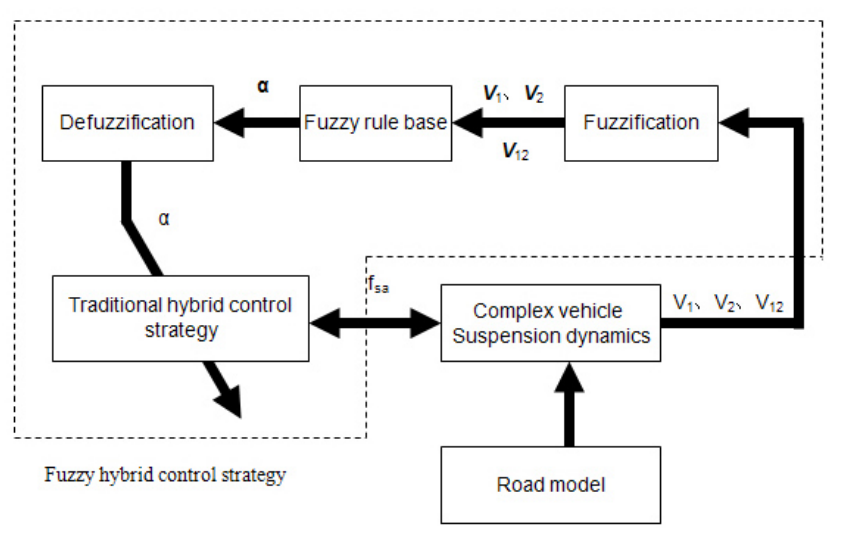

Figure 1. Block diagram of fuzzy hybrid control system.

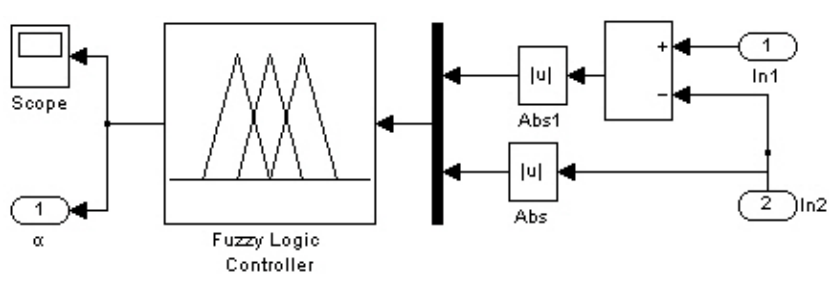

Figure 2. Fuzzy logic control module of the proposed control strategy. 


$$
\boldsymbol{V}_{1}=\{\mathrm{ES}, \mathrm{VS}, \mathrm{SM}, \mathrm{ME}, \mathrm{LA}, \mathrm{VL}, \mathrm{EL}\}
$$$$
V_{12}=\{E S, V S, S M, M E, L A, V L, E L\}
$$

Where, ES = extremely small VS = very small, $\mathrm{SM}=$ small, $\mathrm{ME}=$ medium, $\mathrm{LA}=$ large and $\mathrm{VL}=$ very large, $\mathrm{EL}=$ extremely large. A linguistic output variable is also defined to describe the relative ratio $\alpha$ as follows.

$$
\boldsymbol{\alpha}=\left\{\mathrm{Z}_{1}, \mathrm{Z}_{2}, \mathrm{Z}_{3}, \mathrm{Z}_{4}, \mathrm{Z}_{5}, \mathrm{Z}_{6}, \mathrm{Z}_{7}, \mathrm{Z}_{8}, \mathrm{Z}_{9}\right\}
$$

Where, $Z_{i}(i=1, \ldots, 9)$ are fuzzy variables of $\alpha$, respectively. The membership functions of $\boldsymbol{V}_{1}, \boldsymbol{V}_{12}$ and $\boldsymbol{\alpha}$ are shown in Figs 3 5. Based on expert knowledge, a collection of 49 rules of the following form can be generated.

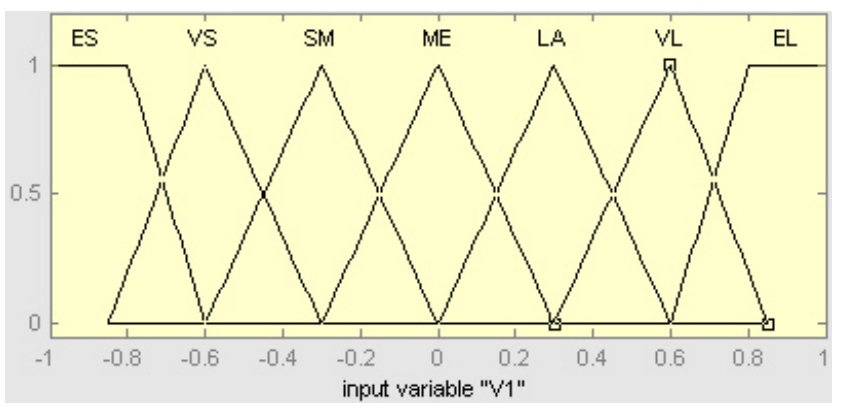

Figure 3. Membership functions of linguistic variable $\boldsymbol{V}_{1}$.

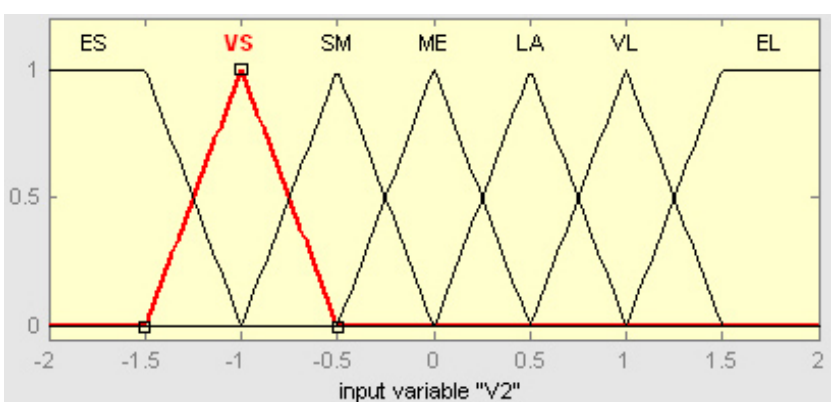

Figure 4. Membership functions of linguistic variable $V_{2}$.

$$
\begin{aligned}
& R^{i}=I F \boldsymbol{V}_{1} \text { IS EL AND } \boldsymbol{V}_{12} \text { IS ES THEN } \boldsymbol{\alpha}=Z 9 \\
& R^{i+1}=I F \boldsymbol{V}_{1} \text { IS ES AND } \boldsymbol{V}_{12} \text { IS EL THEN } \boldsymbol{\alpha}=Z 1 \\
& R^{i+2}=I F \boldsymbol{V}_{1} \text { IS EL AND } \boldsymbol{V}_{12} \text { IS EL THEN } \boldsymbol{\alpha}=Z 5
\end{aligned}
$$

The collection of above fuzzy control rules of the proposed hybrid control strategy can be represented as shown in Table 1. The above control rules can also be visualized as Fig. 6. The center-of-gravity method is employed to infer these fuzzy rules.

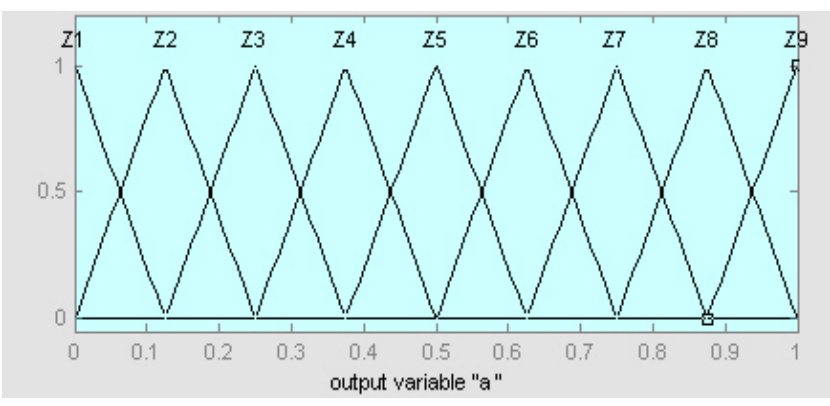

Figure 5. Membership functions of linguistic variable $a$.

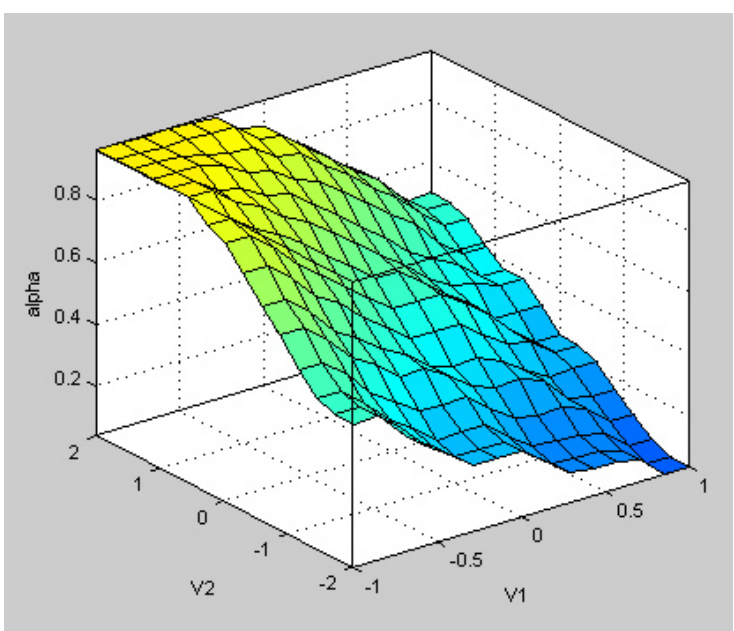

Figure 6. 3D cloud map of fuzzy logic control module including 49 rules.

\section{Table 1. Fuzzy rule base of fuzzy hybrid control strategy}

\begin{tabular}{cllllllll}
\hline V2 & V1 & ES & VS & SM & ME & LA & VL & EL \\
ES & Z5 & Z6 & Z7 & Z8 & Z9 & Z9 & Z9 \\
VS & Z4 & Z5 & Z6 & Z7 & Z8 & Z9 & Z9 \\
SM & Z3 & Z4 & Z5 & Z6 & Z7 & Z8 & Z9 \\
ME & Z3 & Z4 & Z4 & Z5 & Z6 & Z7 & Z8 \\
LA & Z2 & Z3 & Z4 & Z5 & Z5 & Z6 & Z7 \\
VL & Z2 & Z3 & Z3 & Z4 & Z4 & Z5 & Z6 \\
EL & Z1 & Z2 & Z3 & Z3 & Z4 & Z4 & Z5 \\
\hline
\end{tabular}


The proposed fuzzy hybrid control strategy was coded using C language in ColdWarrior 4.6, the damping coefficients of skyhook control and groundhook control are all set to $40 \mathrm{kN} \cdot \mathrm{s} / \mathrm{m}$.

\section{Design of an electronic control unit}

Electronic control units offer a modular, networked approach to real-time vehicle control and diagnostics. For evaluating the performance of the proposed fuzzy hybrid control strategy using HIL technology, a general electronic control unit based on Freescale S12XDP512 microcontroller (Freescale Co. LTD, USA) was designed using Altium Designer 6.9 software (Fig. 7) in order to investigate the influences of time-delay factors on control performance of the proposed control strategy. The developed electronic control unit mainly consists of two power units, a 16-channel analog-to-digital convertor, a 8-channel digital-to-analog convertor and a 4-channel current driver, etc. The fuzzy hybrid control strategy was compiled and then downloaded into the ECU through a background debug module (BDM).

\section{HIL system for verification of fuzzy hybrid control strategy}

Testing electronic control units in real vehicles is a time-consuming, costly process, and comes very late in the automotive development process. To achieve the aimed quality of the newly developed vehicles, electronic control unit's testing has to be conducted as early as possible within the vehicle development process. At pre-

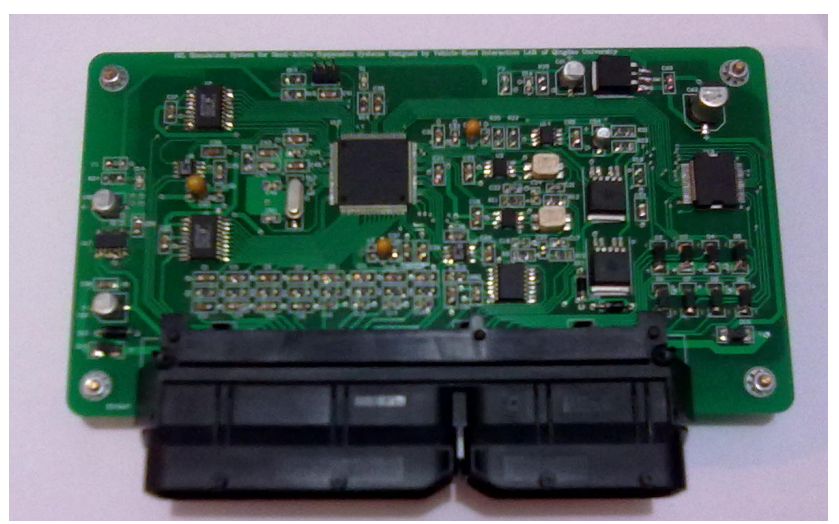

Figure 7. An electronic control unit based on Freescale XDP512 16-bit microcontroller. sent, the traditional methods are increasingly replaced by laboratory tests using HIL technology. HIL is an advanced technique for conducting system-level evaluation of electronic control units in a comprehensive, cost effective, and repeatable manner. In this study, a cost-effective HIL simulation system was designed at the Laboratory of Vehicle-Road Interaction, College of Mechanical \& Electrical Engineering, Qingdao University for verification of the proposed fuzzy hybrid control strategy.

The hardware subsystem of the HIL system was consisted of a PC workstation equipped with a 4-core CPU, a specially-designed general 16-bit ECU, a PCI-1711 multifunctional data acquisition card (ADVANTECH Co. LTD, TAIWAN) and a power supply with an output voltage range of $5 \sim 36 \mathrm{~V}$ (Fig. 8).

The software subsystem of the HIL system mainly includes a quarter car semi-active suspension model designed using MATLAB/Simulink, a road model as well as several I/O ports designed using Real-Time Windows Target Toolbox for interfacing with the developed ECU.

According to Lagrange's equation, the following differential equations of a quarter car model of semi-active suspension systems can be derived,

$$
\begin{aligned}
& m_{s} \ddot{x}_{s}+k_{s}\left(x_{s}-x_{u}\right)-f_{s a}=0 \\
& m_{u} \ddot{x}_{u}+k_{s}\left(x_{u}-x_{s}\right)-k_{t}\left(x_{u}-x_{r}\right)+f_{s a}=0
\end{aligned}
$$

Where $m_{\mathrm{s}}, m_{\mathrm{u}}, k_{\mathrm{s}}, k_{\mathrm{t}}, f_{\mathrm{sa}}, x_{s}, x_{u}, x_{r}, \ddot{x}_{s}$, and $\ddot{x}_{u}$ are sprung mass, unsprung mass, suspension stiffness, tire stiffness, the force generated by a semi-active damper, displacement of the sprung mass, displacement of unsprung mass, road profile, the velocity of sprung mass and the velocity of unsprung mass, respectively (Fig. 9). By defining $\boldsymbol{x}=\left[\begin{array}{lllll}\dot{x}_{S} & \dot{x}_{u} & x_{S} & x_{u} & \dot{x}_{r}\end{array}\right]^{\mathrm{T}}$ as the state vector, the following state equations in matrix form can be obtained.

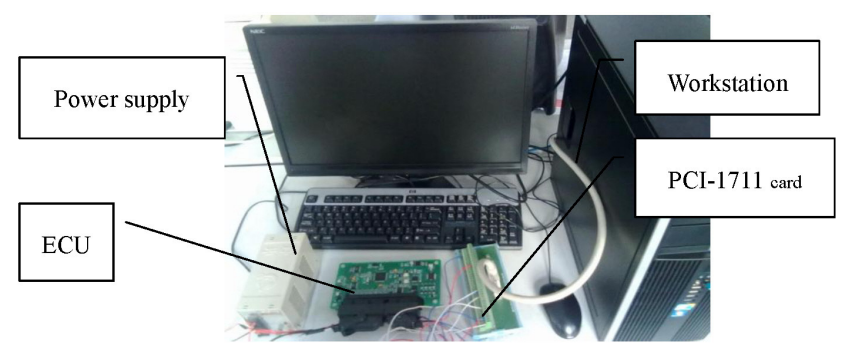

Figure 8. A HIL system for evaluating the proposed control strategy. 


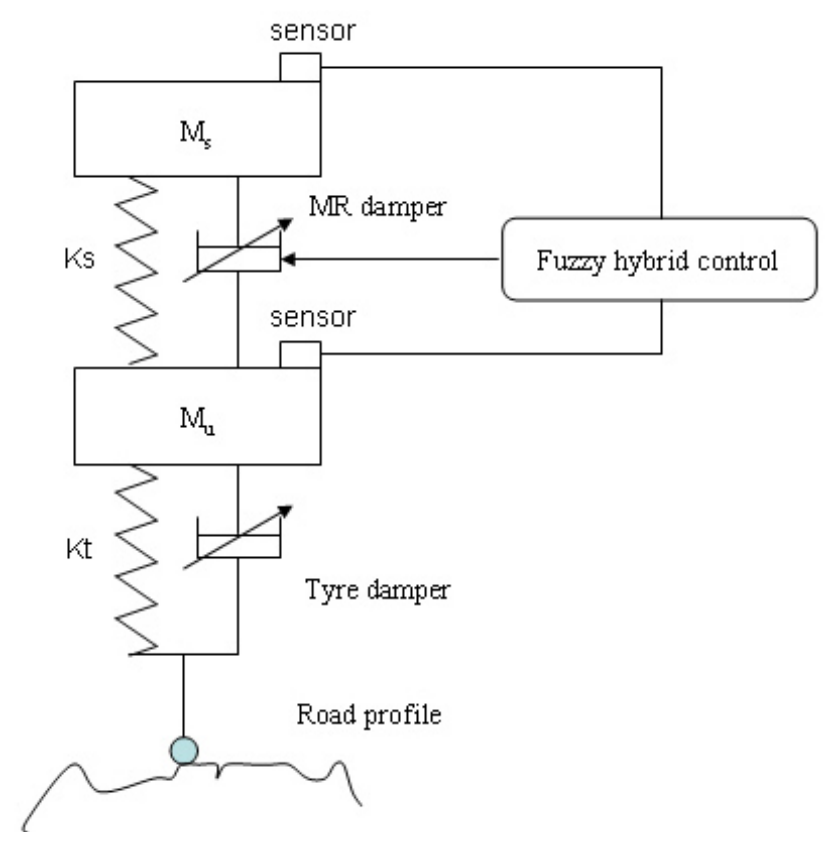

Figure 9. Schematic of a quarter car semi active suspension system.

$$
\begin{aligned}
& \dot{x}=A x+B u \\
& y=C x+D u
\end{aligned}
$$

Where: $\boldsymbol{u}=\left[\mathrm{f}_{\mathrm{sa}} X_{r}\right]^{\mathrm{T}}$ is the input vector, and

$$
\begin{aligned}
\boldsymbol{A} & =\left[\begin{array}{ccccc}
0 & 0 & -\frac{k_{s}}{m_{s}} & \frac{k_{s}}{m_{s}} & 0 \\
0 & 0 & \frac{k_{s}}{m_{u}} & \frac{-k_{s}-k_{t}}{m_{u}} & \frac{k_{t}}{m_{u}} \\
1 & 0 & 0 & 0 & 0 \\
0 & 1 & 0 & 0 & 0 \\
0 & 0 & 0 & 0 & -2 \pi f_{0}
\end{array}\right] \\
\boldsymbol{B} & =\left[\begin{array}{cccc}
\frac{1}{m_{s}} & 0 & \\
-\frac{1}{m_{u}} & 0 & 0 \\
0 & 0 & 0 \\
0 & 0 & 0 \\
0 & 2 \pi \sqrt{G_{0} v_{0}}
\end{array}\right] \quad\left[\begin{array}{lllll}
1 & 0 & 0 & 0 & 0 \\
0 & 1 & 0 & 0 & 0 \\
0 & 0 & 1 & 0 & 0 \\
0 & 0 & 0 & 1 & 0 \\
0 & 0 & 0 & 0 & 1
\end{array}\right] \quad \boldsymbol{D}=\left[\begin{array}{lllll}
0 & 0 & 0 & 0 & 0 \\
0 & 0 & 0 & 0 & 0
\end{array}\right]^{\mathrm{T}} \\
\boldsymbol{C} &
\end{aligned}
$$

Where: $x_{\mathrm{r}}$ is road displacement; $G_{0}$ is the road roughness coefficient; $v_{0}$ is the vehicle speed, $w(t)$ is a Gaussian white noise with mean value of 0 ; $f_{0}$ is the low cut-off frequency. The matrices $\boldsymbol{C}$ and $\boldsymbol{D}$ are specially set to unit matrix and null matrix for extracting the state vector.

The main parameters of the quarter car model are set to $m_{\mathrm{s}}=4500 \mathrm{~kg}, \mathrm{~m}_{\mathrm{u}}=500 \mathrm{~kg}, \mathrm{k}_{\mathrm{s}}=0.2 \mathrm{M} \cdot \mathrm{N} / \mathrm{s}, \mathrm{k}_{\mathrm{t}}=2 \mathrm{M} \cdot \mathrm{N} / \mathrm{s}$. According to the above data, all elements of matrices of $\boldsymbol{A}$, $\boldsymbol{B}, \boldsymbol{C}, \boldsymbol{D}$ can be calculated, respectively.

The road model used in the developed semi-active suspension system is a filtered Gaussian white noise (Yu and Crolla, 2003).

$$
\dot{x}_{\mathrm{r}}(t)=-2 \pi \mathrm{f}_{0} x_{\mathrm{r}}(t)+2 \pi \sqrt{\mathrm{G}_{0} \mathrm{v}_{0}} w(t)
$$

\section{Road-friendliness criterion used in HIL simulation}

Two typical criteria chosen to evaluate road-friendliness of a semi-active suspension system governed by the proposed fuzzy hybrid control strategy: Dynamic Load Coefficient (DLC) and Dynamic Load Stress Factor (DLSF).

DLC overcomes the drawbacks of road- friendliness evaluation based on static load, in which the dynamic interaction between vehicle and road is considered (Sweatman, 1983). This criterion is mainly used to evaluate permanent road damage caused by moving vehicles. DLC is defined as follows.

$$
\text { DLC }=\frac{\text { standard deviation of dynamic load }}{\text { static load }}
$$

The DLC value of a suspension system is closely related to road roughness, the speed and the suspension type of a heavy vehicle.

DLSF was first proposed by K.Yi and K. Hedrick, which is another frequently used road-friendliness criterion [4], DLSF can be expressed as follows.

$$
\mathrm{DLSF}=1+6 \mathrm{DLC}^{2}+3 \mathrm{DLC}^{4}
$$

Where: DLC is the root mean square of dynamic tire force, which can also be calculated using Eq. (7).

\section{HILS methods and main results}

After importing the road model, the semi-active suspension system was linked with the ECU including the 
Yan et al. Road-friendliness of Fuzzy Hybrid Control Strategy Based on Hardware-in-the-Loop Simulations Journal of Biosystems Engineering • Vol. 37, No. 3, 2012 • www.jbeng.org

Table 2. Comparison of dynamic tire force under different control modes

\begin{tabular}{|c|c|c|c|c|}
\hline Items & Road type & Speed, km/h & Passive suspension, $\mathrm{kN}$ & Fuzzy hybrid control strategy in HIL, kN \\
\hline \multirow{4}{*}{$\begin{array}{l}\text { Maximum dynamic } \\
\text { tyre force }\end{array}$} & \multirow{2}{*}{ A } & 90 & 90.776 & 82.394 \\
\hline & & 110 & 102.870 & 94.209 \\
\hline & \multirow{2}{*}{ B } & 90 & 206.340 & 158.680 \\
\hline & & 110 & 216.360 & 191.140 \\
\hline \multirow{4}{*}{$\begin{array}{l}\text { Standard deviation of } \\
\text { dynamic tyre force }\end{array}$} & \multirow{2}{*}{ A } & 90 & 25.237 & 23.250 \\
\hline & & 110 & 26.806 & 25.534 \\
\hline & \multirow{2}{*}{$\mathrm{B}$} & 90 & 51.039 & 47.638 \\
\hline & & 110 & 54.233 & 51.856 \\
\hline
\end{tabular}

Table 3. Road-friendliness of a semi-active suspension system under different control modes

\begin{tabular}{|c|c|c|c|c|}
\hline \multirow{2}{*}{ Road type } & \multirow{2}{*}{ Road-friendliness criterion } & \multirow{2}{*}{ Speed, km/h } & \multicolumn{2}{|c|}{ Road-friendliness } \\
\hline & & & Passive suspension, $\mathrm{kN}$ & Fuzzy hybrid control strategy in HIL \\
\hline \multirow{4}{*}{$A$} & \multirow{2}{*}{ DLC } & 90 & 0.52 & 0.47 \\
\hline & & 110 & 0.55 & 0.52 \\
\hline & \multirow{2}{*}{ DLSF } & 90 & 2.84 & 2.47 \\
\hline & & 110 & 3.09 & 2.84 \\
\hline \multirow{4}{*}{$\mathrm{B}$} & \multirow{2}{*}{ DLC } & 90 & 1.04 & 0.97 \\
\hline & & 110 & 1.10 & 1.06 \\
\hline & \multirow{2}{*}{ DLSF } & 90 & 10.99 & 9.30 \\
\hline & & 110 & 12.65 & 11.53 \\
\hline
\end{tabular}

proposed control strategy. The solver option was set to fixed-step ode45 (Runge-Kutta), real-time workshop's system target file to rtwin.tlc, HIL simulation for investigating on the performance semi-active suspension systems under the control of the proposed strategy was carried out at 90 $\mathrm{km} / \mathrm{h}, 110 \mathrm{~km} / \mathrm{h}$ on A-type and B-type motorways, during which the relative ratio of hybrid control block of the proposed control strategy was intelligently controlled by the proposed fuzzy logic control module. The standard deviations and the maximum values of dynamic tire forces under different control modes are summarized in Table 2 for the following road-friendliness evaluation process. A semi-active suspension system governed by the proposed fuzzy hybrid control strategy suppressed the maximum and the deviation values of the dynamic tire forces when compared with those of a passive suspension system.

Using DLC and DLSF criteria, road-friendliness of a semi-active suspension system and a passive suspension system is shown in Table 3. It is straightforward that a semi-active suspension system under the proposed fuzzy hybrid control strategy improved road-friendliness on both A-type and B-type motorways.

\section{Conclusions}

(1) A fuzzy hybrid control strategy was designed by combining hybrid control strategy and a fuzzy logic control module for improving road-friendliness.

(2) A hardware-in-the-loop (HIL) simulation system was designed to verify the proposed fuzzy hybrid control strategy.

(3) Semi-active suspension system under the control of the fuzzy hybrid control strategy can be utilized to improve road friendliness.

\section{Acknowledgements}

This research was supported by the Scientific Research Foundation for Outstanding Young Scientists of. Shandong Province (No. BS2010ZZ002), Scientific Research Foundation for Returned Overseas Chinese Scholars, State Education Ministry. 


\section{Reference}

Caponetto, R., O. Diamante, G. Fargione, A. Risitano and D. Tringali. 2003. A soft computing approach to fuzzy sky-hook control of semiactive suspension. IEEE Transactions on Control Systems Technology 11(6):786798.

Choi, S. B., H. K. Lee and E. G. Chang. 2001. Field test results of a semi-active ER suspension system associated with skyhook controller. Mechatronics 11(3):345-353.

Goncalves, F. D. 2001. Dynamic Analysis of Semi-Active Control Techniques for Vehicle Applications, M.S. thesis, Virginia Polytechnic Institute and State University, Virginia.

Guglielmino, E., T. Sireteanu, C. W. Stammers, G. Ghita and M. Giuclea. 2008. Semi-active Suspension Control: Improved Vehicle Ride and Road Friendliness, SpringerVerlag London Limited.

Kortüm, W., M. Valášek, Z. Šika, W. Schwartz, P. Steinbauer and O. Vaculín. 2002. Semi-active damping in automotive systems: design-by-simulation. International Journal of Vehicle Design 28(1/2/3):103-120.

Nicolás, C. F., J. Landaluze, E. Castrillo, M. Gastón and R.
Reyero. 1997. Application of fuzzy logic control to the design of semi-active suspension systems. In: The 6th International IEEE Conference on Fuzzy Systems FUZZIEEE'97, Barcelona, Spain.

OECD. 1998. Dynamic interaction between vehicles and infrastructure experiment (DIVINE).

Sweatman, P. F. 1983. A study of dynamic wheel forces in axle group suspensions of heavy vehicles. Special Report, Australian Road Research Board, Report No. SR27.

Valášek, M., W. Kortüm, Z. Šika, L. Magdolen and O. Vaculín. 1998. Development of semi-active road-friendly truck suspensions. Control Engineering 6:735-744.

Yan, T., D. Liu, H. Chen and W. Huo. 2007. Road-friendliness of heavy vehicles based on active suspension systems. Chinese Journal of Mechanical Engineering 43(2):163167 (In Chinese, with English abstract).

Yi, K. and J. K. Hedrick. 1989. Active and semi-active heavy truck suspensions to reduce pavement damage. SAE Technical Paper 892486.

Yu, F. and D. Crolla. 2003. Dynamics and Control of Vehicles. People's Communications Press (In Chinese, with English abstract). 